\title{
Therapeutic equivalence of ivermectin $1 \%$ and two novel formulations combined of ivermectin $1 \%$ + fluazuron $12.5 \%$ for the control of Rhipicephalus (Boophilus) microplus in beef cattle from Uruguay
}

\author{
Diego Robaina, Silvana Alvariza and Gonzalo Suárez* \\ Pharmacology and Therapeutics Unit, Department of Clinics, Veterinary Hospital, Faculty of Veterinary Medicine, \\ University of the Republic, Montevideo, Uruguay
}

\begin{abstract}
Background: Novel combinations of ivermectin (IVM) and fluazuron (FLU) are presented as an alternative for the control of ticks in cattle. Applying a combination of drugs with the aim to affect different stages of the parasite's life cycle is established as a potential measure to achieve the control of ticks in cattle.

Aim: To determine the therapeutic equivalence between two novel formulations of IVM 1\% combined with FLU $12.5 \%$ tested on bovines naturally infested with Rhipicephalus (Boophilus) microplus.

Methods: Forty adult beef cattle were randomized into four groups $(n=10)$ : IVM [1\% $(0.2 \mathrm{mg} / \mathrm{kg})]$, combinations groups A and B [IVM 1\% (0.2 mg/kg) + FLU 12.5\% (2.5 mg/kg), each], and control [untreated]). On days 14, 27, and 49 after administration, the presence of ticks was ranked as null, low, medium, and high; a cumulative link model was adjusted to evaluate treatment response.

Results: Although all groups had some animals with the presence of ticks until day 27, on day 14 IVM [odds ratios (OR) 0.013, CI95\%: 0.001-0.014, $p<0.01$ ], A (OR 0.01, CI95\%: 0.00-0.07, $p<0.01$ ) and B (OR 0.01, CI95\%: $0.00-0.148, p<0.01)$ groups were different when compared to the control group, unlike on day 27 where only groups A (OR 0.02, CI95\%: 0.00-0.17, $<<0.01)$ and $\mathrm{B}$ (OR 0.06, C195\%: 0.00-0.46, $p<0.01$ ) remained different from the control group. On day 49 post-administration, IVM and B did not differ from the control group, with 0.95 probability (CI95\% 0.92-1.02) of high parasite burden. At day 49 post-administration, group A was the only group free of ticks (OR 0.01, CI95\%: 0.00-0.13, $p<0.01$ ).

Conclusions: Pharmacotechnical differences in combined formulations should be considered in therapeutic equivalence studies.
\end{abstract}

Keywords: Bovine, Drug combination, Ectoparasites, Tick.

\section{Introduction}

In livestock systems, parasitic disease is one of the health problems that causes the greatest economic losses (Wolstenholme et al., 2004; Waller, 2006). Ticks are one of the most destructive ectoparasites of livestock in tropical and subtropical areas. They are responsible for severe economic losses through both the direct effects of blood feeding and indirectly as vectors of pathogens (Eckstein et al., 2015; Molento, 2020).

Multiple treatments and strategies have been implemented all around the world when it comes to achieving the control or eradication of Rhipicephalus microplus on field conditions, using different drugs under different formulations applied by different routes, enhancing the fact that there is no unique way to treat $R$. microplus, and making it necessary to adapt the strategy for the different epidemiological situation and risks (Sutherst et al., 1979; George et al., 2004; Ahmed, 2016; Rodriguez-Vivas et al., 2018).
In Uruguay, the level of control of $R$. microplus populations in cattle and the resistance phenomenon are worrying. The main control methods can be classified into chemical methods (application of acaricidal products and insect growth regulators) and nonchemical methods (application of biological products and biological control). Currently, chemical treatments are almost the only available resource for the control of this parasite (Fiel and Nari, 2013). The emergence of parasite populations resistant to chemicals used to treat them, or the resistance mechanisms on new chemical classes, continues to pose a serious threat to stable chemical parasite management strategies (Holdsworth et al., 2006). The first diagnosis of resistance to synthetic pyrethroids in Uruguay was reported in 1994 (Cardozo et al., 1984; Fiel and Nari, 2013). Subsequently in 2006, resistance to the molecule fipronil (Phenylpyrazoles) was officially diagnosed (Cuore et al., 2007), in 2009 to amitraz (Amidines)

*Corresponding Author: Gonzalo Suárez. Unidad de Farmacología y Terapéutica, Departamento de Clínicas y Hospital Veterinario, Facultad de Veterinaria, Universidad de la República, Montevideo, Uruguay. 
(Cuore et al., 2012), and in 2010 to the macrocyclic lactones ivermectin (IVM) and moxidectin (Cuore et al., 2015). Of the active ingredients approved for use in the campaign against $R$. microplus, only fluazuron (FLU) (benzoylurea) has no resistance reports in our country (Cuore et al., 2017). New studies confirm an aggravation of the problem, with different parasitic populations involved in resistance to different active ingredients (Cuore et al., 2016).

In this context, the increasing resistance to treatment procedures calls for changes in the existing parasite control strategies (Waller, 2006; Molento et al. 2011; Molento, 2020), further motivating the rational use of available pharmacological tools. Parasitic drug resistance is the consequence of a genetic modification or an increase in the frequency of expression of a hereditary character. In both cases, certain individuals in a population are given the ability to survive the pharmacological effect of therapeutic concentrations of a drug, in relation to the normal (susceptible) population of the same species (Stone, 1972; Nolan, 1990; Prichard, 1994). The strategic application of effective drugs with different mechanisms of action (i.e., non-neurotoxic drugs along with neurotoxic drugs), adjusted to the prevailing epidemiological conditions for ticks, is one of the possible alternatives when there is resistance to the main groups available. Combining drugs with different mechanisms of actions and effects on different stages of $R$. microplus' life cycle is an alternative for the strategic control of this parasite on cattle (Gomes et al. 2015).

The aim of this study was to determine therapeutic equivalence between two novel formulations of IVM $1 \%$ combined with FLU $12.5 \%$ tested on bovines naturally infested with $R$. (Boophilus) microplus on beef cattle under field conditions in Uruguay.

\section{Materials and Methods}

\section{Farm location}

The experiment was conducted from January to March 2019 in a farm located in Tacuarembó, Uruguay, South

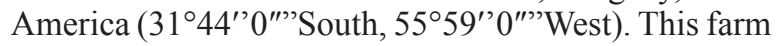
has a history of using IVM, fipronil, or amitraz for the control of $R$. microplus, but has never applied FLU in the rotation between the three drugs mentioned earlier. Experimental design

Forty adult beef cattle (Aberdeen Angus) with a high degree of $R$. microplus infestation were randomized into four different groups $(n=10)$. The groups were as follows: IVM (IVM 1\%, SC, IVOMEC $®$, Boehringer Ingelheim, Mexico); A (Formulation A; IVM 1\% [0.2 $\mathrm{mg} / \mathrm{kg}]+\mathrm{FLU} 12.5 \%$ [2.5 mg/kg], subcutaneous administration (SC)]; B (Formulation B; IVM 1\% [0.2 $\mathrm{mg} / \mathrm{kg}]+\mathrm{FLU} 12.5 \%$ [2.5 mg/kg], SC); and control (untreated tracer bovines, as true indicators of tick populations in the field). Groups A and B and IVM received treatments on day 0 via a single dose through $\mathrm{SC}$ injection in the neck, following the recommendations stated by the manufacturer. The dose was not repeated at any moment. All formulations have been approved as Animal Drugs Product from Ministerio de Agricultura y Pesca in Uruguay. Commercial names of both formulations $\mathrm{A}$ and $\mathrm{B}$ have been omitted for ethical reasons. All animals were kept in the same pasture during the entire experiment.

\section{Data recovery}

Animals were checked for the presence of ticks on the day of administration (day 0 ) and follow-up was carried out on days 14,27, and 49 after administration. On each day mentioned, the number of adult engorged female ticks (EFT) (4.5-5 $\mathrm{mm}$ of diameter) was estimated by manually counting the number of engorged females on four body zones: perianal, flank, neck, and face, as recommended by the WAAVP (Holdsworth et al., 2006). Four ranks were built in view of the presence of EFT: null (no presence of EFT), low (1-50) (less than 50 EFT), medium (50-100) (between 50 and 100 EFT), and high (>100) (more than 100 EFT). The trial ended on day 49 after the administration due to the explicit request from the owner of the farm to retake the usual strategy for the control of $R$. microplus.

Since we had three different treatments to compare with, at different time periods, we decided to evaluate the presence of EFT in the animals into three time periods according to the duration of the tick's parasitic cycle (mode $=23$ days) (Fiel and Nari, 2013): early response (treatment response on day 14 post-treatment), late response (treatment response on day 27 posttreatment), and delayed response (treatment response on day 49 post-administration).

\section{Statistical analysis}

In every period, we compared all treatments against the control group. Ranked data [null, low (1-50), medium $(50-100)$, and high $(>100)]$ as the outcome variables were analyzed by cumulative link models $(\mathrm{clm})$ for ordinal data using the ordinal package for R Statistical Software (version 4.0.3 [2020-10-10]) (R Studio Core Team, 2020). The best-fitted $\mathrm{clm}$ was selected by the goodness of fit criteria and AIC. The final model represents the presence of adult $R$. microplus for each treatment applied on cattle in different time periods; time (days) was handled as a continuous variable and group as a categorical variable (control, IVM, A, and B). Estimated odds ratios (OR) and 95\% confidence intervals $(95 \% \mathrm{CI})$ over cut-offs of the outcome scale were calculated. A $p$-value $<0.05$ was considered statistically significant.

\section{Ethical approval}

The present study was approved by the Comisión Ética en Uso de Animales (CEUA) of Facultad de VeterinariaUniversidad de la República, under protocol No. CEUA FVET-PI 506/17.

\section{Results}

No adverse effects or signs of systemic intoxication were observed in the experimental animals after the 
administration of treatments. Moreover, no cattle died and no concomitant medications were administered during these experiments.

Distribution of the presence of EFT found on each day for each treatment is shown in Figure 1. Data are expressed as a probability of infestation for every treatment at each moment.

On day 0 , the data analysis of the treated groups did not show differences in the burden of $R$. microplus when compared to the control group, with a high probability of the presence of EFT from 0.80 to 1.0 between IVM, $\mathrm{A}, \mathrm{B}$, and control groups $(p>0.05)$. One of the strengths of this study was the fact that the control group always showed threshold coefficients of "medium" and "high" $>0.70$. During the whole study, no animals in the control group were ranked with the null presence of ticks, evidencing a continuous exposition of cattle to these parasites. The effect of time on the presence of EFT in the control group was not significant during the entire study (day 0-49) (OR 0.97, CI95\%: 0.93-1.01, $p=0.148)$.

\section{Early response}

Early response from the data analysis for the IVM group shows the effect on the $R$. microplus burden when compared to the control group. On day 14 postadministration, the $\mathrm{clm}$ displayed a favorable treatment response in the IVM group. The odds of the presence of EFT on day 14 was 0.013 times compared to the control group of animals, holding constant all other variables (OR 0.013, CI95\%: 0.001-0.014, $p<0.01$ ). The probability that the IVM group presents medium or high EFT versus low EFT is 0.20 (CI95\%: 0.05-0.53) plogis, with no probability to null (no EFT presence in animals).

For the combined formulations of IVM and FLU, similar results were obtained. On day 14 postadministration, the odds of EFT presence was 0.01 (OR 0.01, CI95\%: 0.00-0.07, $p<0.01$ ) and 0.01 (OR 0.01, CI95\%: 0.00-0.148, $p<0.01$ ) (A and $\mathrm{B}$, respectively) times compared to the control group of animals, holding constant all other variables. The probability that the A and B group presents medium or high EFT versus low ticks was 0.10 (CI95\%: 0.02-0.39) and 0.20 (CI95\%: 0.05-0.54) plogis, respectively. This reflects the early response of the IVM plus FLU on both novel formulations combined, with no distinctive effect or favorable response to treatment between any of the formulations applied.

\section{Late response}

Data on response to treatment show differences when compared to the results for the early response. On day 27 post-administration, the probability that the IVM group continues to present medium or high EFT versus low EFT is 0.17 (CI95\%: 0.04-0.64) plogis. An OR of 0.17 (CI95\%: 0.04-0.50) for the null presence of EFT revealed a continuous challenge from the tick parasites. The odds of the presence of ticks on day 27 was not statistically different when compared to the control group, holding constant all other variables (OR 0.30, CI95\%: 0.04-1.86, $p=0.196$ ).

$A$ and $B$ groups did not differ on the results obtained for the late response data. The odds for EFT presence on day 27 were 0.02 times for A (OR 0.02, CI95\%: 0.00$0.17, p<0.01)$ and 0.06 times for B (OR 0.06, CI95\%:

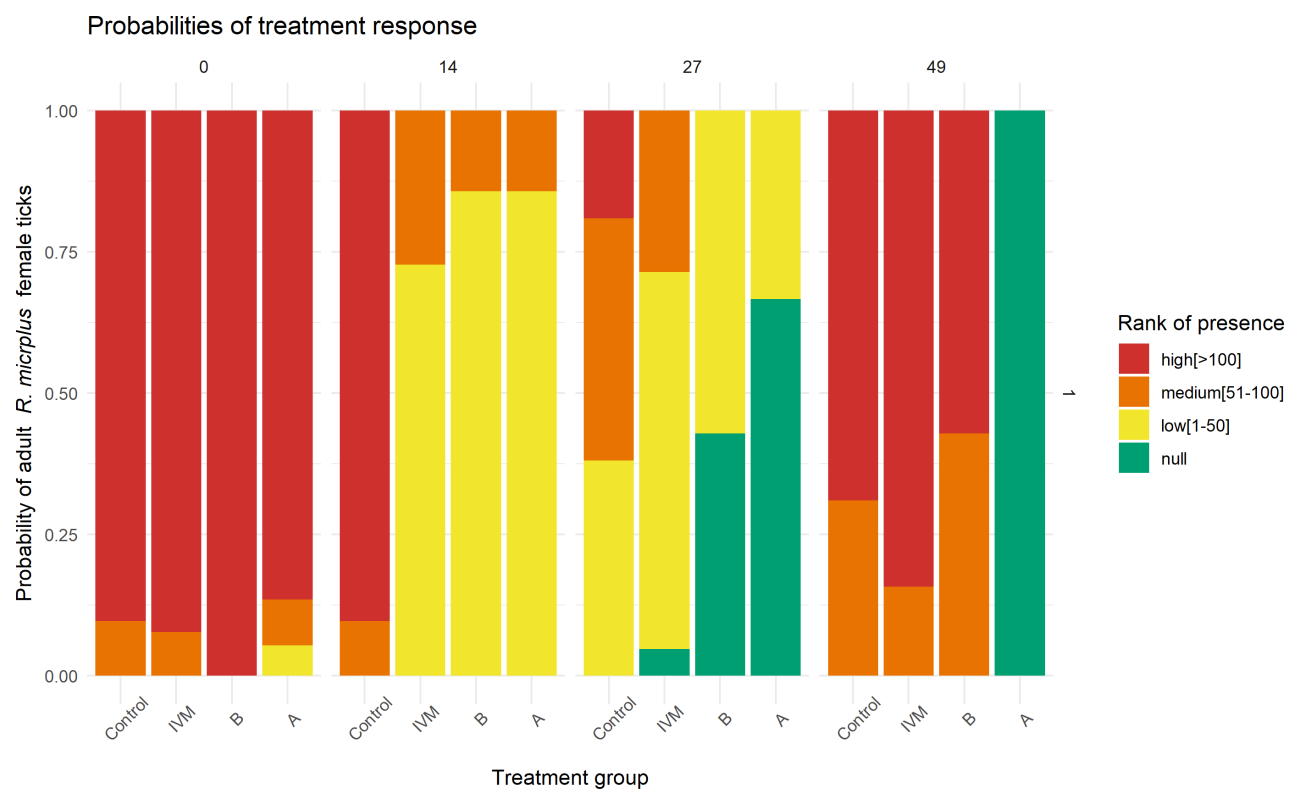

Fig. 1. The presence of adult female ticks on cattle under field conditions. IVM: group treated with IVM $(0.2 \mathrm{mg} / \mathrm{kg}, 1 \%$, subcutaneous); (A and B) two novel formulations of IVM combined with FLU (IVM 1\% [0.2 mg/kg] + FLU 12.5\% [2.5 mg/kg], subcutaneous); control group acted as tracer cattle (untreated). Days after formulations administration: 0, 14, 27 , and 49. 
$0.00-0.46, p<0.01)$ when compared to the control group animals, holding constant all other variables. The probability of null the presence of EFT was 0.74 (CI95\% 0.42-0.92) and $0.50(0.22-0.78)$ for groups A and $\mathrm{B}$ for the same time period when compared to the control group, respectively.

\section{Delayed response}

IVM group on day 49 post-administration showed similar behavior as the control group (OR 2.09, CI95\%: $0.32-13.3, p=0.43$ ), with a probability of 0.95 (CI95\% 0.92-1.02) animals with high parasite burden (more than 100 EFT), revealing a lack of protection in the IVM group after 49 days of administration.

Nevertheless, combined formulations showed differences in the delayed control of adult female ticks. For group A, all animals were free of EFT (OR 0.01, CI95\%: 0.00-0.13, $p<0.01$ ), while animals in group B showed the presence of EFT (OR 1.04, CI95\%: 0.18$5.80, p=0.96)$, compared to the control group animals, holding constant all other variables. Both combined formulations of IVM and FLU achieved different levels of control over tick population.

\section{Discussion}

For each time frame, we established treatment success (favorable treatment response) according to the probability of diminishing the level of infestation between the treated group and the control group. With ranked data and probabilities, we approached an epidemiological understanding: tick infestation under field conditions is a matter of probability of contact between the larvae and the host.

\section{Early response}

The probability for the early response of IVM reflects what has been proven by various authors on the rapid efficacy of IVM on the R. microplus population. Davey et al. (2005) reported an $83.2 \%$ reduction in adult female $R$. microplus after a single dose administration of IVM $1 \%$ on cattle after 12 days of administration. Cruz et al. (2015) tested different formulations of IVM administered by different routes, and under field conditions in Brazil. For subcutaneous administration, the maximum efficacy was observed on day 14 posttreatment $(89.8 \%$ ) for a $0.2 \mathrm{mg} / \mathrm{kg}$ dose of IVM.

The fact that some parasites remained on the animals might be due to the life cycle of $R$. microplus and the relationship between IVM plasma concentration and the life cycle (and hence the daily blood intake of the ticks), as exposed by Davey et al. (2005). A favorable treatment response with the null presence of EFT was not achieved on any of the animals included in the study after 14 days post-administration, compatible with an active parasite population as it was expected when working on field conditions and natural infestation.

\section{Late response}

Our results obtained for IVM on late response are consistent with the ones reported by Cruz et al. (2015), where they found that after the administration of an
IVM $1 \%$ formulation $(0.2 \mathrm{mg} / \mathrm{kg})$, the efficacy dropped from $90 \%$ (14 days post-administration) to a range of $32 \%-75 \%$ on day 28 post-administration. The high variability reported by the authors mentioned is due to the different strains of tick populations that can be found when working under field conditions.

When comparing these results to the ones obtained after applying IVM under different formulations, Cruz et al. (2015) reported an efficacy higher than 90\% between days 7 and 49 post-treatment when working with an IVM $3.15 \%(0.63 \mathrm{mg} / \mathrm{kg})$ long-acting formulation. The late response observed with long-acting formulations could be explained by the distinctive plasma profile of IVM obtained with these depot formulations. Lifschitz et al. (2007) demonstrated a higher persistence for IVM $3.15 \%$ formulations compared to IVM 1\%, which could have significant effects on parasite control and dosage needed for control/eradication. Davey et al. (2010) proposed a cut-off point $(8 \mathrm{ng} / \mathrm{ml})$ for IVM plasma concentration administered in "long-acting" or "reservoir" formulations, above which there is efficacy against $R$. microplus.

Efficacy trials for IVM $3.15 \%$ after subcutaneous administration provide results that support the premise raised by Lifschitz et al. (2007) on the persistence of action based on the plasma profile. Nava et al. (2019) reported efficacy profiles against infestations with $R$. microplus after the administration of IVM $3.15 \%$; from day 7 post-treatment until day 21 , they observed efficacy percentages close to $100 \%$. These results respond to a longer exposure time of the ticks to concentrations of IVM that are lethal for the parasite, affecting different evolutionary stages that appear with the advance of the parasitic biological cycle.

For combined formulations, the results show that both novel formulations of IVM combined with FLU for subcutaneous administration behave the same way for the 27 days of administration under field conditions. These field efficacy results are consistent with the fact that both formulations behaved as bioequivalent when it comes to IVM (Robaina et al., 2021) and no major differences should be expected for both early and late response treatment success.

\section{Delayed response}

The response for the IVM group observed 49 days post-administration of a single dose of IVM $(0.2 \mathrm{mg} /$ $\mathrm{kg}$ ) is consistent with the results reported by Davey et al. (2010) and Pereira (2009) for persistence of IVM efficacy on $R$. microplus, where treatment with IVM should be applied on a 30-day interval to achieve a successful control of ticks.

Group A showed a better performance when compared to group B, as shown by the lower OR on EFT presence after 49 days of administration. Group A achieved 100\% efficacy despite the fact of an active population of $R$. microplus (showed by the result in the control group). This could be related to the long-term control effect of FLU included in the combination. FLU is responsible 
of inhibiting chitin synthesis on immature ticks, preventing them to achieve adult stages. Differences found for both subcutaneous combinations of IVM and FLU could be related to differences between formulations (drug-drug interaction) and the impact that this could bring to the pharmacokinetic profile, as stated for different veterinary formulations studied by Toutain et al. (1997).

Insect growth regulators, such as FLU, could be responsible for the improvement of favorable treatment response achieved at day 49 by the combination of IVM and FLU given the delayed effect that this type of compounds have on insects' life cycle (Holdsworth et al., 2006), when compared to a single subcutaneous administration of IVM $(0.2 \mathrm{mg} / \mathrm{kg})$. Gomes et al. (2015) tested different FLU presentations for the control of $R$. microplus; they tested a subcutaneous injection of FLU combined with IVM $(1.6 \mathrm{mg} / \mathrm{kg}$ FLU $+0.63 \mathrm{mg} / \mathrm{kg}$ IVM) which achieved a lesser degree of protection compared to a pour-on formulation of FLU $(2.5 \mathrm{mg} / \mathrm{kg})$ or even versus a subcutaneous injection of IVM $(0.63 \mathrm{mg} / \mathrm{kg})$. Gomes et al. (2015) report an $82 \%$ reduction in adult ticks on day 21 post-administration of a combined formulation of IVM and FLU. When compared to the results obtained with the subcutaneous administration of IVM $(0.63 \mathrm{mg} / \mathrm{kg})$ that reached a peak of $86 \%$ efficacy on day 21 post-administration and the FLU group (pour-on administration, $2.5 \mathrm{mg} / \mathrm{kg}$ ) which showed more than $90 \%$ efficacy between days 14 and 49 post-administration.

The lower number of ticks on days 27 and 49 for the control group could be a result of environmental effects (rain and heat), but mostly because the probability of picking up larvae from the pasture was lower than the reason being that all animals were kept in the same pasture. As the treatments were showing efficacy, the number of adult ticks was dropping. Efficacy field studies should be carried out to shed knowledge on the strategic use of combined formulations and to compare the efficacy of IVM and FLU under different formulations. Further studies are needed on the pharmacokinetic profile of combined formulations to better understand the interchangeability in pharmaceutical alternatives of combined IVM and FLU.

\section{Conclusion}

The combined formulations of IVM and FLU showed differences in efficacy and persistence under field conditions of its component drugs, which made it more effective than treatment with a single drug in controlling tick population in field conditions. The question is whether the use of combined formulations is the correct way or if the focus should be placed on improving the strategic use of these drugs. Complete control or eradication of parasite ticks could be achieved following a specific strategy and adjusting the time between doses.

\section{Acknowledgment}

This study was funded by ANII (Agencia Nacional de Investigación e Innovación) under a project from "Fondo María Viñas Modalidad I".

\section{Conflict of interest}

The authors declare that there is no conflict of interest. Authors'contributions

GS conceived, designed, and supervised the project. DR and GS executed the experiment and analyzed the data. All the authors interpreted the data, wrote, and critically revised the manuscript for intellectual content and approved the final version.

\section{References}

Ahmed, N.A. 2016. Review of economically important cattle tick and its control in Ethiopia. Adv. Life Sci. Technol. 42, 51-64.

Cardozo, H., Nari, A., Franchi, M., López, A. and Donatti, N. 1984. Estudio sobre la ecología del Boophilus microplus en tres áreas enzoóticas del Uruguay. Veterinaria (Montevideo) 20, 4-10.

Cruz, B.C., Lopes, W.D., Maciel, W.G., Felippelli, G., Fávero, F.C., Teixeira, W.F., Carvalho, R.S., Ruivo, M.A., Colli, M.H., Sakamoto, C.A., da Costa, A.J. and De Oliveira, G.P. 2015. Susceptibility of Rhipicephalus (Boophilus) microplus to ivermectin $(200,500$ and $630 \mu \mathrm{g} / \mathrm{kg})$ in field studies in Brazil. Vet. Parasitol. 207(3-4), 309-317.

Cuore, U., Acosta, W., Bermúdez, F., Da Silva, O., García, I., Pérez Rama, R., Luengo, L., Trelles, A. and Solari, M.A. 2015. Tratamiento generacional de la garrapata. Aplicación de una metodología en un manejo poblacional para la erradicación de Rhipicephalus (Boophilus) microplus resistentes a lactonas macrocíclicas. Veterinaria (Montevideo) 51(198), 14-25.

Cuore, U., Altuna, M., Cicero, L., Fernández, F., Luengo, L., Mendoza, R., Nari, A., Pérez Rama, R., Solari, M.A. and Trelles, A. 2012. Aplicación del tratamiento generacional de la garrapata en la erradicación de una población multirresistente de Rhipicephalus (Boophilus) microplus en Uruguay. Veterinaria (Montevideo) 48, 5-13.

Cuore, U., Solari, M.A. and Trelles, A. 2017. Current status of resistance and first diagnostic of multiple resistance Rhipicephalus (Boophilus) microplus tick simultaneously resistant to five drugs in Uruguay. Veterinaria (Montevideo), 53(205), 13-19.

Cuore, U., Solari, M.A., Piaggio, J., Chelle, B., Di Rienzo, D., Machado, N., Politi, P., Trelles, A. and Rampoldi, O. 2016. Comportamiento biológico y farmacocinético de dos formulaciones comerciales de ivermectina $3,15 \%$ en bovinos. Veterinaria (Montevideo) 52(201), 13-22.

Cuore, U., Trelles, A., Sanchis, J., Gayo, V. and Solari, M.A. 2007. Primer diagnóstico de resistencia al Fipronil en la garrapata común del ganado Boophilus microplus. Veterinaria (Montevideo) 42, 35-41. 
Davey, R.B., Miller, J.A., George, J.E. and Miller, R.J. 2005. Therapeutic and persistent efficacy of a single injection treatment of ivermectin and moxidectin against Boophilus microplus (Acari: Ixodidae) on infested cattle. Exp. App. Acarol. 35(1-2), 117129.

Davey, R.B., Pound, J.M., Miller, J.A. and Klavons, J.A. 2010. Therapeutic and persistent efficacy of a long-acting (LA) formulation of ivermectin against Rhipicephalus (Boophilus) microplus (Acari: Ixodidae) and sera concentration through time in treated cattle. Vet. Parasitol. 169(1-2), 149-156.

Eckstein, C., Lopes, L., Romero Nicolino, R., Oliveira, C.S. and Haddad, J. 2015. Economic impacts of parasitic diseases in cattle. CAB Rev. Perspect. Agric. Vet. Sci. Nutr. Nat. Resour. 10(51), 1; doi:10.1079/PAVSNNR201510051

Fiel, C. and Nari, A. 2013. Enfermedades parasitarias de importancia clínica y productiva en rumiantes. Montevideo, Uruguay: Hemisferio Sur.

George, J., Pound, J. and Davey, R. 2004. Chemical control of ticks on cattle and the resistance of these parasites to acaricides. Parasitology, 129(S1), S353-S366.

Gomes, L.V., Lopes, W.D., Cruz, B.C., Teixeira, W.F., Felippelli, G., Maciel, W.G., Bichuette, M.A., Ruivo, M.A., Alcantara Colli, M.H., Carvalho, R.S., Martinez, A.C., Soares, V.E. and da Costa, A.J. 2015. Acaricidal effects of fluazuron $(2.5 \mathrm{mg} /$ $\mathrm{kg}$ ) and a combination of fluazuron (1.6 mg/ $\mathrm{kg})+$ ivermectin $(0.63 \mathrm{mg} / \mathrm{kg})$, administered at different routes, against Rhipicephalus (Boophilus) microplus parasitizing cattle. Exp. Parasitol. 153, $22-28$

Holdsworth, P.A., Kemp, D., Green, P., Peter, R.J., De Bruin, C., Jonsson, N.N., Letonja, T., Rehbein, S., Vercruysse, J. and World Association for the Advancement of Veterinary Parasitology. 2006. World association for the advancement of veterinary parasitology (W.A.A.V.P.) guidelines for evaluating the efficacy of acaricides against ticks (Ixodidae) on ruminants. Vet. Parasitol. 136(1), 29-43.

Lifschitz, A., Virkel, G., Ballent, M., Sallovitz, J., Imperiale, F., Pis, A. and Lanusse, C. 2007. Ivermectin $(3.15 \%)$ long-acting formulations in cattle: absorption pattern and pharmacokinetic considerations. Vet. Parasitol. 147(3-4), 303-310.

Molento, M. 2020. Avaliação seletiva de bovinos para o controle do carrapato. Brasilia, Brasil.: Ministério da Agricultura, Pecuária e Abastecimento. Available via https://www.gov.br/agricultura/pt-br/ assuntos/producao-animal/arquivos-publicacoesbem-estar-animal/CARRAPATOS2.pdf (Accesed 20 November 2020).
Molento, M.B., Fortes, F.S., Pondelek, D.A., Borges, F.D., Chagas, A.C., Torres-Acosta, J.F. and Geldhof, P. 2011. Challenges of nematode control in ruminants: focus on Latin America. Vet. Parasitol. 180(1-2), 126-132.

Nava, S., Toffaletti, J.R., Morel, N., Guglielmone, A.A. and Mangold, A.J. 2019. Efficacy of winter-spring strategic control against Rhipicephalus (Boophilus) microplus infestations on cattle in an area with ecological conditions highly favourable for the tick in northeast Argentina. Med. Vet. Entomol. 33(2), 312-316.

Nolan, J. 1990. Acaricide resistance in single and multihost tick and strategies for control. Parassitologia $32,145-153$.

Pereira, J.R. 2009. The efficiency of avermectins (abamectin, doramectin and ivermectin) in the control of Boophilus microplus, in artificially infested bovines kept in field conditions. Vet. Parasitol. 162(1-2), 116-119.

Prichard, R. 1994. Anthelmintic resistance. Vet. Parasitol. 54, 259-268.

R Studio Core Team. 2020. R: a language and environment for statistical computing. Vienna, Austria: R Foundation for Statistical Computing. Available via https://www.R-project.org/

Robaina, D., Alvariza, S. and Suárez, G. 2021. Bioequivalence of two novel formulations of ivermectin $1 \%$ combined with fluazuron $12.5 \%$ for subcutaneous administration in cattle. J. Pharm. Pharmacogn. Res. 9(1), 88-97.

Rodriguez-Vivas, R.I., Jonsson, N.N. and Bhushan, C. 2018. Strategies for the control of Rhipicephalus microplus ticks in a world of conventional acaricide and macrocyclic lactone resistance. Parasitol. Res. $117,3-29$.

Stone, B.F. 1972. The genetics of resistance by ticks to acaricides. Aust. Vet. J. 48, 345-350.

Sutherst, R.W., Norton, G.A., Barlow, N.D., Conway, G.R., Birley, M. and Comins, H. N. 1979. An analysis of management strategies for cattle tick (Boophilus microplus) control in Australia. J. Appl. Ecol. 16(2), 359.

Toutain,P.L., Upson, D.W., Terhune, T.N. and McKenzie, M.E. 1997. Comparative pharmacokinetics of doramectin and ivermectin in cattle. Vet. Parasitol. 72(1), 3-8.

Waller, P.J. 2006. Sustainable nematode parasite control strategies for ruminant livestock by grazing management and biological control. Anim. Feed Sci. Tech. 126, 277-289.

Wolstenholme, A.J., Fairweather, I., Prichard, R., von Samson-Himmelstjerna, G. and Sangster, N.C. 2004. Drug resistance in veterinary helminths. Trends Parasitol. 20(10), 469-476. 